\title{
Dead Sea Minerals-Induced Positive Stress as an Innovative Resource for Skincare Actives
}

\author{
Meital Portugal-Cohen ${ }^{1,2 *}$, Maria F. Dominguez ${ }^{3}$, Miriam Oron ${ }^{1,2}$, Robert Holtz ${ }^{4}$, \\ Ze'evi Ma'or'1,2 \\ ${ }^{1}$ Ahava-Dead Sea Laboratories, Lod, Israel \\ ${ }^{2}$ Dead Sea and Arava Science Center, The Laboratory for Skin Biochemistry and Biotechnology, Ein Gedi, Israel \\ ${ }^{3}$ Lonza-Personal Care, South Plainfield, USA \\ ${ }^{4}$ Biolnnovation Laboratories Inc., Lakewood, USA \\ Email: ${ }^{*}$ meital.p@ahava.com
}

Received 20 January 2015; accepted 8 February 2015; published 11 February 2015

Copyright @ 2015 by authors and Scientific Research Publishing Inc.

This work is licensed under the Creative Commons Attribution International License (CC BY).

http://creativecommons.org/licenses/by/4.0/

(c) (i) Open Access

\section{Abstract}

Objective: Exposure to certain stresses in small doses might lead to a protective effect by improving resistance to other stressors. Dead Sea (DS) minerals can be a relevant source to induce positive stress due to their high salinity and unique mineral combination. This concept could be further optimized using advanced unique cell biotechnology. The purpose of this study was to elucidate the innovative concept of DS minerals (water extract and black mud) supplementation in small amount to Pichia pastoris yeast growth media as a positive stress by testing the capability of accepted fermentation compounds to affect the appearance of skin. Methods: Skin equivalents were topically applied with different Pichia pastoris fermentations (Metabiotics ${ }^{\mathrm{TM}}$ ). Skin elasticity biomarkers were tested, since loss of elasticity and suppleness is a natural skin aging process leading to deeper wrinkles and loss of firmness. A preliminary screening at the gene level using DNA microarray was performed and subsequently, the following proteins were detected using ELISA or immunoblotting assays: elastin, fibulin-1, lysyl oxidase (LOX), metalloproteinase 3 (MMP-3), E-cadherin, claudin 4, tight junction protein (TJP)-1 and TJP-2. UVB irradiation was selected as a stressor. Results: Fermentation compounds generated in the presence of small doses of DS minerals affected the expression of various elasticity-related genes in skin. Moreover, they significantly attenuated the abnormal UVB-induced alterations, the proteins elastin, fibulin-1, LOX, MMP-3, E-cadherin and TJP-2. Conclusions: The observations clearly demonstrate that when DS Metabiotics ${ }^{\mathrm{TM}}$ compounds are topically applied, significant alterations in several biomarkers that contribute to skin elasticity occur. Thus, these novel compounds have the potential to serve as skincare actives.

${ }^{*}$ Corresponding author. 


\section{Keywords}

\section{Skin Elasticity Biomarkers, Skin Aging, Active Ingredients, Dead Sea Minerals, Positive Stress}

\section{Introduction}

\subsection{Positive Stress and Hormesis}

Stressors, both endogenous and exogenous, are encountered constantly through an organism's life cycle. As a first line of defense against external insults, skin frequently works to protect itself and to effectively shield the internal tissues against threats such as, UV irradiation, free radicals attack and exposure to environmental agents. Over time, damage to the skin occurs, which leads to drastic changes in skin's integrity and to the activation of aging processes. Researchers from various scientific disciplines such as toxicology, medicine and cosmetology have studied the effects of skin exposure to various chemicals stressors at different concentrations at the tissue, organ and whole organism level [1]. Major advances have been made in screening and evaluation processes which help to determine more accurately the damaging effects of substance exposure (chemicals or other stressors) on skin health [2]. Following dose-response work, the phenomenon of "hormesis" was proposed, in which a damaging substance surprisingly elicited a positive effect when used in small concentrations [1]. Extensive research has been carried out exploring hormetic effects of a wide range of substances, ranging from antioxidants, to biological intermediates etc. [2].

\subsection{Dead Sea Environment as a Positive Stressor}

The Dead Sea (DS), although famous worldwide for its healing properties, is a stressful living environment: DS water and mud with their almost saturated natural salinity prevents organisms, such as fish and aquatic plants, from living in these conditions. At the same time, in spite of their living restrictions, it is known that DS water and mud can be therapeutic for different skin and arthritis disorders in moderate doses of exposure [3].

DS water, as compared to seawater, contains a tenfold concentration and an unusual composition of various cations; mainly magnesium, sodium, calcium, potassium and strontium. These metal cations are balanced with halogen anions mainly chlorides and bromides [4]. DS black mud, which contains antibacterial and hyperemic properties, is rich in minerals similarly to DS water [5]. The therapeutic properties of DS water and mud have been thoroughly investigated and published. Clinical studies demonstrate that DS water and mud contribute to a significant improvement in patients' symptoms of skin disorders, mainly psoriasis, atopic dermatitis, seborrheic dermatitis and vitiligo [6]-[8].

Recently, it has been demonstrated that photo-damage protective effects occur in human skin organ culture following exposure to DS water extract or DS mud at certain doses [9]-[12].

Due to its extreme and unusual conditions, it is well-known that the DS area contains unique flora and botanicals which have adaptogenic characteristics [4] [12]. This allows an organism to pre-adapt itself in a manner that contributes to its survival against various endogenous stressors (aging and different pathological processes) and exogenous stressors (extreme conditions such as radiation, temperature, dryness, etc.). Secondary metabolites consist of enzymes, vitamins, and various natural components are presented in the extract and can contribute to protection against various stressors [13]. Thus, here DS-induced stress has a positive effect by producing secondary metabolites enabling better coping with harsh environmental conditions. Indeed, DS region-originated botanical extracts, such as palm date (Phoenix dactilifera) extracts, Dunaliella salina green algae extract and Jujube (zyziphus) extracts, were reported to have beneficial skin healing effects.

\subsection{Metabiotics ${ }^{\mathrm{TM}}$ Technique Using DS Minerals as a Positive Stress for Pichia pastoris Yeast Growth}

DS water's and DS mud's potency to induce positive stress was assumed due to their high salinity, unique mineral combination and well established therapeutic effects. Based on hormesis concepts, we investigated how their positive stress could be further optimized using advanced unique cell biotechnology, Metabiotics ${ }^{\mathrm{TM}}$ (international patent application is WO2010/011885 A1). In this respect, DS water extract and DS mud were provided 
as nutrients in small doses for the yeast Pichia pastoris (P. pastoris), which utilized and completely metabolized them as positive stress supplement by fermentation. Hence, this fermentation product might have biological effects on skin and serve as a potent skincare active. In this work, DS minerals supplementation as a positive stress to $P$. pastoris was examined by testing the effect of fermentation extracts on 3D human skin equivalents.

\section{Materials and Methods}

\subsection{DS Water Extract and DS Mud Supplementation to Pichia pastoris (P. pastoris)}

\subsubsection{Materials}

P. pastoris was obtained from ATCC (\# 60372) and maintained on yeast-peptone-dextrose (YPD) agar plates. The culture is grown in shaker flasks containing YPD liquid media. The fermentation media is Yeast NitrogenBase (YNB) growth media supplemented with glycerol, containing $2.7 \% \mathrm{H}_{3} \mathrm{PO}_{4}, 0.09 \% \mathrm{CaSO}_{4}, 1.8 \% \mathrm{~K}_{2} \mathrm{SO}_{4}$, $1.5 \% \mathrm{MgSO}_{4}, 0.41 \% \mathrm{KOH}, 4 \%$ glycerol (Sigma St. Louis, MO), $1 \%$ and $2 \%$ sterile Dead Sea water extract (Osmoter ${ }^{\mathrm{TM}}$ ), $1 \%$ and $2 \%$ sterile Dead Sea Mud (Ahava-Dead Sea Laboratories, Inc., Israel). Antifoam sigmaemulsion B was used at onset of fermentation only (Sigma, St. Louis, MO).

\subsubsection{Fermentation}

P. pastoris cultures maintained on YPD agar plates were grown in YPD liquid media at $30^{\circ} \mathrm{C}, 250 \mathrm{rpm}$, for 24 hours, until Optical Density (OD) reaches 2.0 at $600 \mathrm{~nm}$. Scale up into 2L bioreactor (2L New Brunswick Scientific, Edison NJ) using Yeast Nitrogen-Base (YNB) growth media and supplemented with glycerol and 1.0\% sterile DS Mud after 24 hours post-inoculation with P. pastoris. The $\mathrm{pH}$ was kept constant at $5.0 \pm 0.5$ with $2 \mathrm{M}$ $\mathrm{NH}_{4} \mathrm{OH}$. The dissolved oxygen (DO) levels were maintained at $30 \%$ saturation by regulating the agitation between 100 and $600 \mathrm{rpm}$. The air flow into the vessel was maintained at $1 \mathrm{VVM}$, throughout the process. The fermentation continued until OD reaches 2.5 at $600 \mathrm{~nm}$, approximately 50 hours post inoculation. At this time the fermentation was considered complete and the batch was harvested and processed.

\subsection{Skin Tissue Preparation}

Upon arrival, the tissues were stored at $4^{\circ} \mathrm{C}$ until used. For use, the tissues were removed from the agaroseshipping tray and placed into a 6-well plate containing $2 \mathrm{ml}$ of assay medium and incubated at $37^{\circ} \mathrm{C} \pm 2^{\circ} \mathrm{C}$ and $5 \% \pm 1 \% \mathrm{CO}_{2}$ overnight.

\subsection{Treatment of the Tissues}

After the initial overnight incubation, the tissues were treated topically with the test materials or phosphate buffered saline (PBS) for untreated controls for 24 hours. At the end of this incubation period the test material was rinsed from the tissues using PBS and then selected tissues were exposed to $225 \mathrm{~mJ} / \mathrm{cm}^{2} \mathrm{UVB}$. Immediately after the UVB exposure the test material or PBS was reapplied to the tissues and the tissues were incubated further for 48 hours. Two sets of tissues were prepared and treated for this study. At the end of this second incubation period, the tissues were washed again and the first set of tissues was used to assess changes in viability (MTT assay), the second set was used for immunoblotting assays. Media from the second set of tissues was used for the ELISA assays and the lysyl oxidase activity assay.

\subsection{Skin Viability by MTT Assay}

After the final wash the tissue culture media was replaced with $2 \mathrm{ml}$ of tissue culture media supplemented with 1 $\mathrm{mg} / \mathrm{ml} \mathrm{MTT}$ and the tissues were incubated for $3 \mathrm{hr}$ at $37^{\circ} \mathrm{C}+2^{\circ} \mathrm{C}$ and $5 \% \pm 1 \% \mathrm{CO}_{2}$. After the incubation with the MTT solution the tissues were rinsed and placed into wells containing $4 \mathrm{ml}$ of isopropyl alcohol to extract the purple formazin crystals. The extraction was allowed to continue overnight, after which $200 \mu \mathrm{l}$ of the isopropyl extracts was transferred to a 96-well plate and the plate was read at $540 \mathrm{~nm}$ using isopropyl alcohol as a blank.

\subsection{DNA Micro-Array for Gene Expression}

Total RNA was isolated using Ambion RNAqueous Kit. mRNA amplification was performed by Ambion Mes- 
sageAmp aRNA kit which included first and second strand cDNA synthesis, cDNA purification and in-vitro transcription to synthesize aRNA and aRNA purification. aRNA was labeled with fluorescent dyes using PerkinElmer ASAP RNA Labeling Kit and concentrated by Molecular Probes Ribogreen Assay.

To purify the labeled aRNA, a Millipore Microcon YM-30 filter column was inserted into a collection tube and filled with $400 \mu \mathrm{l}$ of TE buffer. The Cy3 and Cy5 probes were combined and then added to the Microcon filter and thoroughly mixed with the TE buffer. The filter was centrifuged at 12,000 RPM for 8 minutes and the flow through was discarded. The column was then washed twice with $400 \mu \mathrm{l}$ of TE buffer, discarding the flow though after each centrifugation (12,000 RPM for 8 minutes). After the final wash the filter column was inverted, placed into a new collection tube and centrifuged at 12,000 RPM for 2 minutes to collect the probe.

Microarray Hybridization and Washing was performed by Agilent Technologies Microarrays. The microarrays were then scanned with an Axon GenePix 4100A Scanner with the scanning resolution set to $5 \mu \mathrm{m}$ and analyzed with GenePix Pro software. During the initial scan the PMT gains for the scanner were adjusted such that the Cy5/Cy3 image count ratios were between 0.95 and 1.05 .

\subsection{Determination of Elastin Level by Competitive ELISA}

Soluble $\alpha$-elastin was dissolved in $0.1 \mathrm{M}$ sodium carbonate at a concentration of $1.25 \mu \mathrm{g} / \mathrm{ml}$. $150 \mu \mathrm{l}$ of this solution was then applied to the wells of a 96-well Maxisorp Nunc plate and the plate was incubated overnight at $4^{\circ} \mathrm{C}$. On the following day the wells were saturated with PBS containing $0.25 \%$ bovine serum albomine (BSA) and $0.05 \%$ Tween 20 . The plate was then incubated with this blocking solution for 1 hour at $37^{\circ} \mathrm{C}$ and then washed two times with PBS containing 0.05\% Tween 20.

An anti-elastin antibody solution was prepared (the antibody was diluted 1:100 in PBS containing $0.25 \%$ BSA and $0.05 \%$ Tween 20 ) and $20 \mu \mathrm{l}$ of the solution was added to the tube. The tubes were then incubated overnight at $4^{\circ} \mathrm{C} \pm 2^{\circ} \mathrm{C}$. On the following day, $150 \mu \mathrm{l}$ was transferred from each tube to the 96-well elastin ELISA plate, and the plate was incubated for $1 \mathrm{hr}$ at room temperature. The plate was then washed 3 times with PBS containing $0.05 \%$ Tween 20 . After washing, $200 \mu \mathrm{l}$ of a solution containing a peroxidase linked secondary antibody diluted in PBS containing 0.25\% BSA and 0.05\% Tween 20 was added, and the plate was incubated for $1 \mathrm{hr}$ at room temperature. After washing the plate three times as described above, $200 \mu \mathrm{l}$ of a substrate solution was added and the plate was incubated for 10 to 30 minutes in the dark at room temperature. After this final incubation the plate was read at $460 \mathrm{~nm}$ using a plate reader.

\subsection{Lysyl Oxidase Activity Assay (Abcam Lysyl Oxidase Activity Assay Kit, Red Fluorescence)}

For this assay, $50 \mu 1$ of each cell culture media was added to the wells of a 96-well plate, followed by the addition of $50 \mu \mathrm{l}$ of reaction mix (fluorescent substrate and horse radish peroxidase, mixed in lysyl oxidase assay buffer). The plate was then incubated at $37^{\circ} \mathrm{C}$ for 30 minutes. At the end of the incubation period the plate was read using a fluorometer with an excitation wavelength of $540 \mathrm{~nm}$ and an emission wavelength of $590 \mathrm{~nm}$. Enzyme activity was measured in relative fluorescence units.

\subsection{Quantification of MMP-3 and Fibulin-1 Levels by ELISA Procedure}

\subsubsection{Tissue Preparation for ELISA}

The ELISA plates were prepared by diluting the appropriate capture antibody in PBS. Next, $100 \mu \mathrm{l}$ of the diluted capture antibody was added to the wells of a 96-well ELISA plate and the plate was incubated overnight at room temperature. On the following day the plate was washed three times with $300 \mu \mathrm{l}$ wash buffer $(0.05 \%$ Tween 20 in PBS) and then blocked by adding $300 \mu \mathrm{l}$ of blocking buffer (1\% BSA in PBS) to each well. The plate was incubated with the blocking buffer for at least one hour. After the incubation the blocking buffer was removed and the plate was washed three times as described above.

\subsubsection{MMP-3 Levels by ELISA Procedure}

After sample incubation the plate was washed three times as described above. Once the last wash was removed, $100 \mu \mathrm{l}$ of a biotin conjugated detection antibody was added. After incubating the plate for two hours at room temperature the plate was washed again as described above. $100 \mu \mathrm{l}$ of HRP-streptavidin was then added to each 
well and the plate was incubated for 20 minutes at room temperature. Once the last wash was removed, $100 \mu$ of substrate solution (hydrogen peroxide + tetramethylbenzidine as a chromagen) was added to each well. Once a sufficient level of color development had occurred, $50 \mu \mathrm{l}$ of stop solution ( $2 \mathrm{~N}$ sulfuric acid) was added to each well and the plate was read at $460 \mathrm{~nm}$ using a Packard Spectra Count plate reader.

\subsubsection{Fibulin-1 ELISA Procedure}

A series of standards were prepared and $100 \mu \mathrm{l}$ of each of these standards was dispensed into two wells (duplicates) in the appropriate 96-well plate. Subsequently, $100 \mu \mathrm{l}$ of each sample was added to additional wells and the plate was incubated for two hours at $37^{\circ} \mathrm{C}$. After the incubation the plate was aspirated but not washed, and then $100 \mu \mathrm{l}$ of a biotin conjugated detection antibody was added. After incubating the plate for one hour at $37^{\circ} \mathrm{C}$ the plate was washed three times with wash buffer. $100 \mu \mathrm{l}$ of HRP-streptavidin was then added to each well and the plate was incubated for one hour at $37^{\circ} \mathrm{C}$. Once the last wash was removed, $100 \mu$ l of substrate solution (hydrogen peroxide + tetramethylbenzidine as a chromagen) was added to each well. Once a sufficient level of color development had occurred, $50 \mu \mathrm{l}$ of stop solution (2 N sulfuric acid) was added to each well and the plate was read at $460 \mathrm{~nm}$ using a Packard Spectra Count plate reader.

\subsection{Quantification of E-Cadherin, Z0-1, Z0-2 by Immunoblotting}

\subsubsection{Tissue Preparation for Immunoblotting}

At the end of the treatment period the tissues were homogenized in $500 \mu$ of CelLytic MT Cell Lysis Reagent supplemented with protease inhibitors. The homogenates were then centrifuged at 13,500 RPM ( $\left.4^{\circ} \mathrm{C}\right)$ for 10 min and the supernatant was retained. Prior to use, the protein concentration was determined using a BCA protein assay.

\subsubsection{Bicinchoninic Acid (BCA) Protein Assay}

Fifty volumes of Reagent A (BCA solution) was combined with 1 volume of Reagent B (4\% (w/v) CuSO $\mathrm{Cu}_{4}-5$ $\mathrm{H}_{2} \mathrm{O}$ ) in a 15-ml centrifuge tube. Two hundred microliters of this combined reagent was then be dispensed into a 96-well plate. Next, $10 \mu \mathrm{l}$ of each of the standards or sample was added to respective wells (standards were made using $2 \mathrm{mg} / \mathrm{ml}$ bovine serum albumin dissolved in PBS, and a series of 50\% dilutions were made). The plate was then covered and incubated it at $37^{\circ} \mathrm{C} \pm 2^{\circ} \mathrm{C}$ for $30 \pm 5 \mathrm{~min}$ and then read at $540 \mathrm{~nm}$ using a microplate reader.

\subsubsection{Immunoblotting}

A membrane was equilibrated in PBS and assembled into a Bio-Dot microfiltration apparatus. After assembly, $200 \mu \mathrm{l}$ of PBS was added to each well used in the Bio-Dot and the vacuum was applied to ensure that there was adequate flow through all of the wells. Next, each sample (approximately $10 \mu \mathrm{g}$ ) was assigned a well in the apparatus and was applied to the appropriate well. The samples were filtered under low vacuum. PBS was added to wells not assigned a sample to ensure that the membrane did not dry out during the procedure. At the end of the blotting procedure an additional $200 \mu \mathrm{l}$ of PBS was applied and filtered through each well. The membrane was then removed from the Bio-Dot apparatus, washed in PBS for 5 - 10 minutes and then placed into blocking solution (PBS, 1\% BSA) and allowed to incubate for at least 1 hour at room temperature on a rocking platform.

After blocking, the membrane was transferred to $20 \mathrm{ml}$ of PBST (PBS with $0.1 \%$ Tween-20) and 0.5\% BSA with an appropriate dilution of detection antibody and allowed to incubate overnight at $4^{\circ} \mathrm{C}$ on a rocking platform. After this incubation the membrane was washed 3 times ( $1 \times$ for 15 minutes and $2 \times$ for 5 minutes) in PBST. The secondary antibody (conjugated with a fluorophore) was then incubated with the membrane in $15 \mathrm{ml}$ of PBST with $0.5 \%$ BSA for 1 hour at room temperature and then washed 3 times with PBS (1× for 15 minutes, $2 \times$ for 5 minutes). After the final wash, the membrane was placed into a BioRad Molecular Imager FX and scanned using an excitation laser and emission filter combination appropriate for the fluorophore. Images produced by the scanner were then analyzed using ImageJ image analysis software.

\subsection{Data Analysis}

Values are expressed as mean \pm standard deviation. Differences between average values were tested for significance using the Student t-test and considered significant for $p \leq 0.05$. Each experiment included four 
repetitions.

\section{Results}

P. pastoris fermentation with DS water extract or DS mud was completed successfully. Thus, the fermentation extracts were utilized for a further analysis by application to skin equivalents.

Skin equivalents were topically treated with $2 \%$ of different Metabiotics ${ }^{\mathrm{TM}}$ preparations: $P$. pastoris ferment as a vehicle, DS water extract (Osmoter ${ }^{\mathrm{TM}}$ ) ferment, DS mud ferment and DS mud ferment at 1:1 ratio. For analyzing skin viability, MTT mitochondrial activity assay was used. Skin viability was not changed following topical application of all tested preparations (Figure 1). Thus, all preparations were further analyzed for different proteins playing an important role in skin elasticity, as loss of elasticity and suppleness is a natural skin aging process leading to deeper wrinkles and loss of firmness [14]. These proteins were selected based on a previous micro-array gene analysis on skin equivalents showing that Metabiotics ${ }^{\mathrm{TM}}$ preparations may alter gene expression-related to extracellular matrix (ECM) formation and cell to cell adhesion.

Table 1 describes several elasticity-related genes following the different treatments.

Elasticity-related protein expression or activity was detected following topical application with the different DS ferments. Skin elasticity is impaired as a result of aging or photo-damage and elasticity proteins can be expressed abnormally due to UVB irradiation. Hence, ECM proteins and adhesion dermal-epidermal junction proteins were tested with and without UVB irradiation.

Elastin, fibulin-1, lysyl oxidase (LOX) and metalloproteinase 3 (MMP-3) play an important role in ECM structure. Elastin level significantly increased by $75 \%$ following skin UVB irradiation (Figure 2). This increase was eliminated by pre-treatment with most of the different DS ferments. Elastin level was the most similar to the basal level (control, non-irradiated) following application of DS mud ferment. Pre-treatment with a mixture of DS water extract and DS mud led to a decrease of elastin levels, below the original non-irradiated baseline levels. In non-irradiated skin samples, elastin levels were significantly elevated by $34 \%$ following application of DS mud ferment.

Fibulin-1 is a secreted glycoprotein that is found in association with extracellular matrix structures including elastin-containing fibers. Fibulin-1 levels significantly increased following UVB irradiation more than two-fold (Figure 3). Pre-treatment with DS mud and DS water extract ferments attenuated this elevation.

Lysyl oxidase (LOX) is an extracellular enzyme that catalyzes the oxidation of lysine residues in collagen and elastin precursors resulting in cross-linking of collagen and elastin. This function is essential for stabilization of collagen fibrils and for the integrity and elasticity of mature elastin in skin. LOX activity significantly increased following UVB irradiation by $46 \%$. Among the treatments, only P. pastoris ferment demonstrated a significant increase in LOX activity by $58 \%$ in non-irradiated skin. All three DS ferments significantly abolished UVBinduced LOX activity (Figure 4).

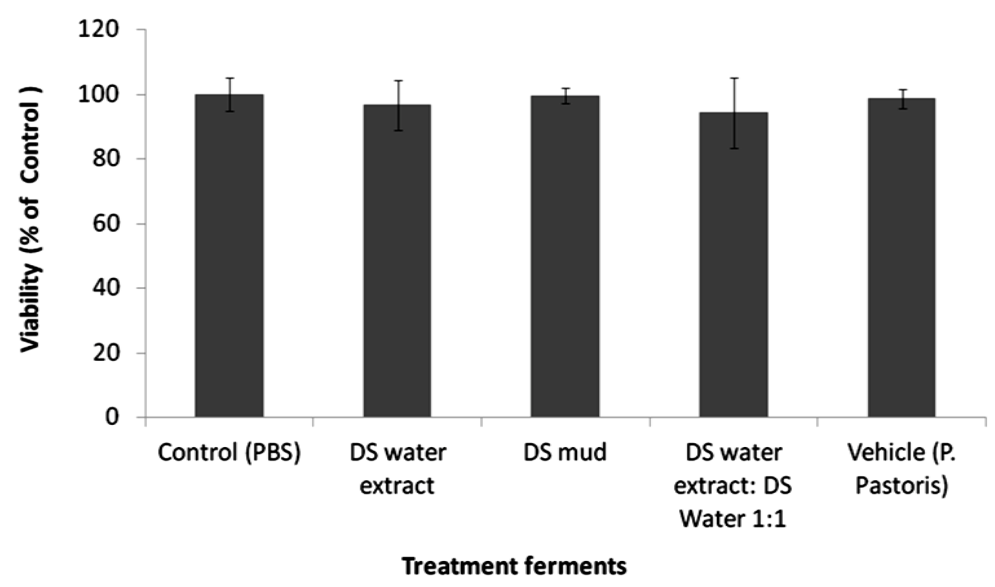

Figure 1. Skin viability following treatment with different $P$. pastoris ferments. MatTek full thickness skin tissues were treated with different $P$. pastoris ferments for $72 \mathrm{hr}$ and their viability was tested by MTT assay as described in the methods section. Data are presented as mean \pm SD. 


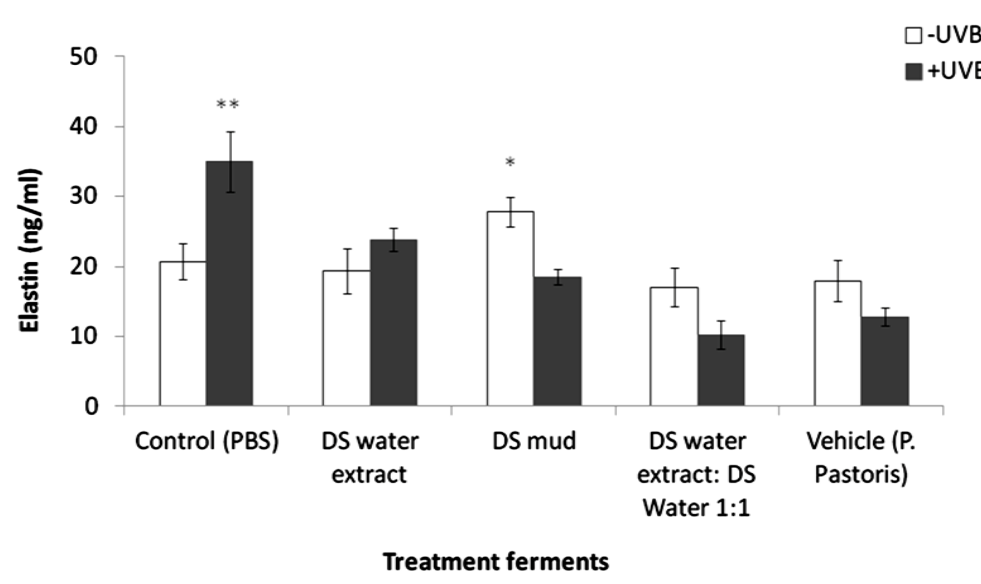

Figure 2. Skin elastin levels following treatment with different $P$. pastoris ferments. MatTek full thickness skin tissues were treated with different $P$. pastoris ferments for $24 \mathrm{hr}$ and UVB irradiated at $225 \mathrm{~mJ} / \mathrm{cm}^{2}$ after the removal of preparations. Immediately after the UVB exposure the test materials were reapplied to the tissues and the tissues were incubated for 48 hours. Elastin levels were tested by ELISA as described in the methods section. Data are presented as mean \pm SD. ${ }^{* *} p<0.01$ irradiated vs. non-irradiated-control; ${ }^{*} p<0.05$ DS mud ferment vs. control-non-irradiated, irradiated vs. non-irradiated-DS water extract:DS mud ferment $1: 1$.

Table 1. Selected elasticity-related genes expression following different $P$. pastoris fermentation preparations.

\begin{tabular}{|c|c|c|c|c|}
\hline \multirow{2}{*}{ Gene name } & \multicolumn{4}{|c|}{ Gene expression vs. control (PBS) } \\
\hline & $2 \%$ P. pastoris ferment & $\begin{array}{l}\text { 2\% DS water extract } \\
\text { ferment }\end{array}$ & $2 \%$ DS mud ferment & $\begin{array}{c}1 \% \text { DS water extract ferment }+ \\
1 \% \text { DS mud ferment }\end{array}$ \\
\hline Elastin & NC & NC & NC & + \\
\hline Fibulin-1 & NC & + & + & + \\
\hline Lysyl oxidase & NC & NC & NC & + \\
\hline MMP-3 & - & - & - & - \\
\hline E-cadherin & NC & + & + & + \\
\hline Claudin-4 & NC & + & + & + \\
\hline TJ protein 1 & + & NC & + & + \\
\hline TJ protein 2 & NC & NC & NC & + \\
\hline
\end{tabular}

*Ratio of median: +: $>1.3$ significant up-regulation; $-:<0.7$ significant down-regulation; NC = no change.

MMP-3 is an enzyme involved in the breakdown of extracellular matrix and is known to increase following irradiation. MMP-3 levels were significantly increased by $11 \%$ following irradiation in the control group. This elevation was eliminated following topical application of the different ferments. In non-irradiated samples, a significant increase of MMP-3 by 12\% was observed following application of DS water extract ferment (Figure 5).

E-cadherin, Claudin 4, TJP-1 and TJP-2 play a significant role in dermal epidermal junction structure.

E-cadherin is a calcium dependent protein and is important in cell adhesion, forming adherent junctions to bind cells within tissues together. E-cadherin levels declined by 34\% following irradiation. Pre-treatment with DS water extract ferment and a mixture of DS water and mud ferments attenuated this reduction. In non-irradiated samples, treatment with DS mud ferment significantly decreased E-cadherin levels by 30\% (Figure 6).

Claudin 4 is an integral membrane protein, which is an important component of the cell junctions, which form the paracellular barrier. With regard to Claudin 4 expression, neither treatment with the ferments nor the 


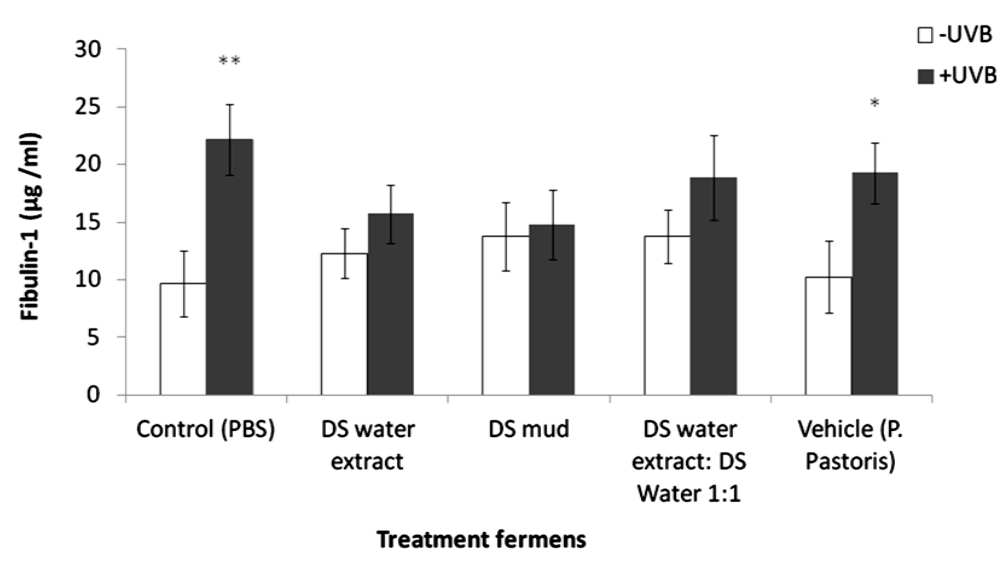

Figure 3. Skin fibulin-1 levels following treatment with different $P$. pastoris ferments. MatTek full thickness skin tissues were treated with different $P$. pastoris ferments for $24 \mathrm{hr}$ and UVB irradiated at $225 \mathrm{~mJ} / \mathrm{cm}^{2}$ after the removal of preparations. Immediately after the UVB exposure the test materials were reapplied to the tissues and the tissues were incubated for 48 hours. Fibulin-1 levels were tested by ELISA. Data are presented as mean \pm SD. ${ }^{* *} p<$ 0.01 irradiated vs. non-irradiated-control; ${ }^{*} p<0.05$ irradiated vs. non-irradiated-vehicle.

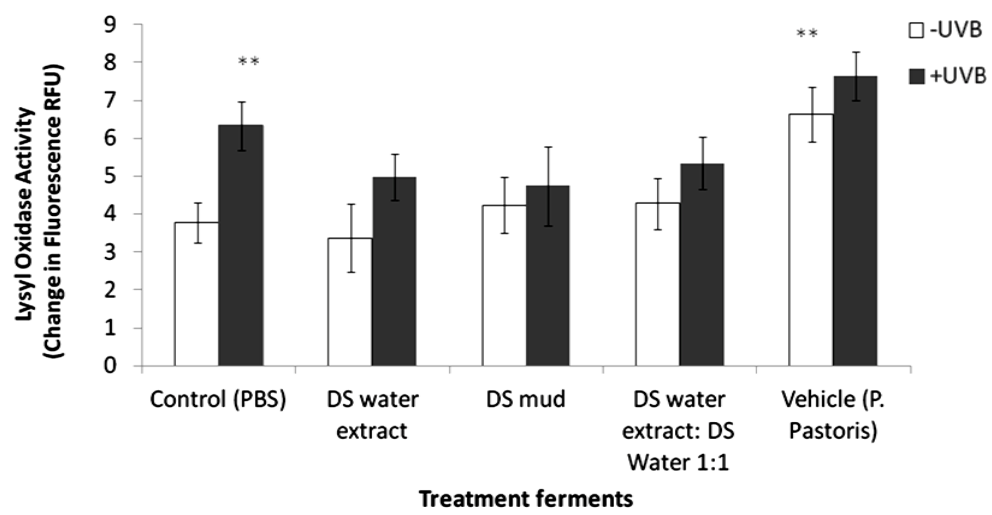

Figure 4. Skin lysyl oxidase (LOX) activity following treatment with different $P$. pastoris ferments. MatTek full thickness skin tissues were treated with different $P$. pastoris ferments for $24 \mathrm{hr}$ and UVB irradiated at $225 \mathrm{~mJ} / \mathrm{cm}^{2}$ after the removal of preparations. Immediately after the UVB exposure the test materials were reapplied to the tissues and the tissues were incubated for 48 hours. LOX activity was tested by Lysyl Oxidase Activity Assay Kit $(\mathrm{ABCAM})$ as described in the methods section. Data are presented as mean \pm SD. ${ }^{* *} p<0.01$ irradiated vs. non-irradiated-control; vehicle vs. control-nonirradiated.

combined UVB exposure and ferments were observed to have an impact. Claudin 4 expression was not observed to be significantly different with any of the treatments (Figure 7).

TJP-1, 2 are involved in signal transduction at cell-cell junctions, anchoring strands of the cytoskeleton and are located on the peripheral membrane.

TJP-1 levels significantly increased by 47\% following UVB irradiation. All ferment treatments reduced this elevation. In non-irradiated skin, vehicle ferment significantly increased TJP-1 levels by $24 \%$ compared to control (Figure 8).

Contrary to TJP-1, TJP-2 levels significantly decreased by 35\% following UVB irradiation. Only pre-treatment with DS water + DS mud ferment attenuated this decrease. In non-irradiated skin TJP-2 decreased by 24\% following application of vehicle ferment (Figure 9). 


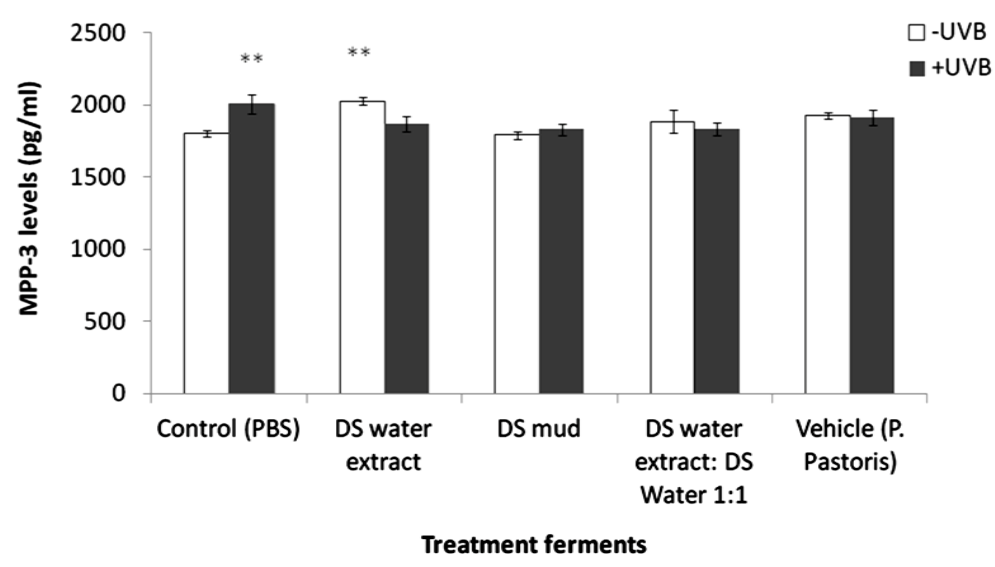

Figure 5. Skin matrix metalloproteinase 3 (MMP-3) levels following treatment with different $P$. pastoris ferments. MatTek full thickness skin tissues were treated with different $P$. pastoris ferments for $24 \mathrm{hr}$ and UVB irradiated at $225 \mathrm{~mJ} / \mathrm{cm}^{2}$ after the removal of preparations. Immediately after the UVB exposure the test materials were reapplied to the tissues and the tissues were incubated for 48 hours. MMP-3 levels were tested by ELISA as described in the methods section. Data are presented as mean \pm SD. ${ }^{* *} p<0.01$ irradiated vs. non-irradiated-control; DS water extract ferment vs. control-non-irradiated.

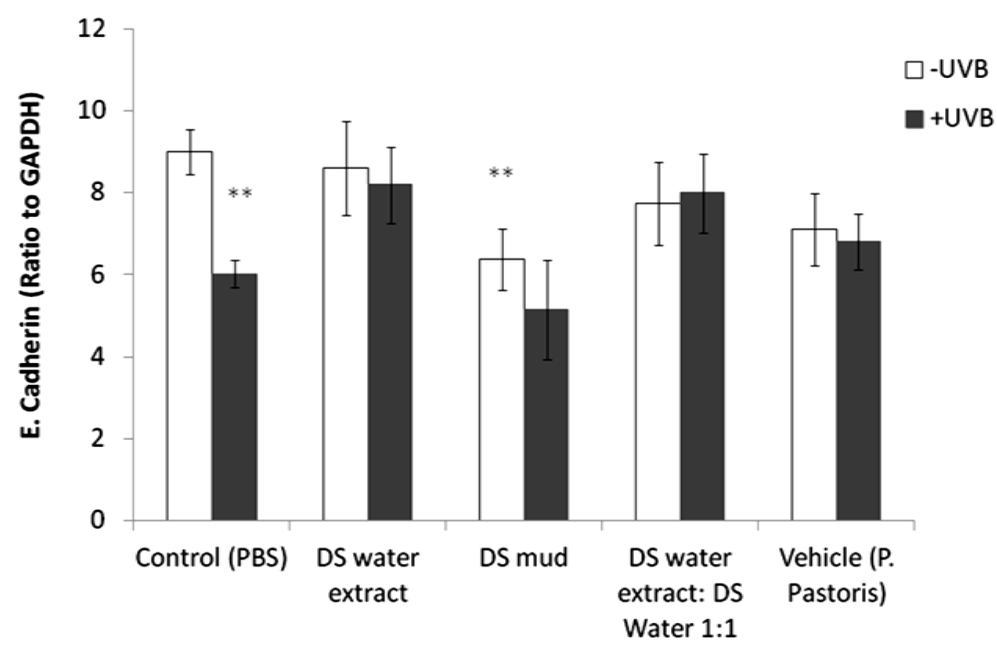

Treatment ferments

Figure 6. Skin E-cadherin levels following treatment with different $P$. pastoris ferments. MatTek full thickness skin tissues were treated with different $P$. pastoris ferments for $24 \mathrm{hr}$ and UVB irradiated at $225 \mathrm{~mJ} / \mathrm{cm}^{2}$ after the removal of preparations. Immediately after the UVB exposure the test materials were reapplied to the tissues and the tissues were incubated for 48 hours. E-cadherin levels were tested using immunoblotting as described in the methods section. Data are presented as mean \pm SD. ${ }^{* *} p<0.01$ irradiated vs. nonirradiated-control; DS mud ferment vs. control-non-irradiated.

\section{Discussion}

Different mechanisms to cope with stressors have evolved in organisms. Interestingly, exposure to certain stresses in small doses might lead to protective effects by improving resistance to other exogenous and endogenous stressors. Thus, positive stress can be beneficial for well-functioning and survival of cells and organisms. 


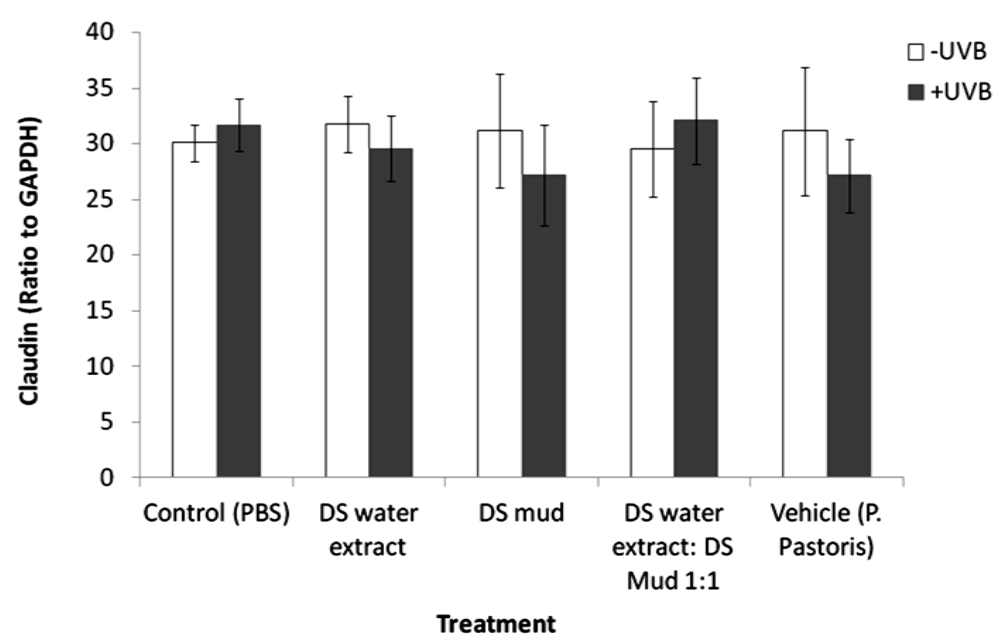

Figure 7. Skin claudin 4 levels following treatment with different $P$. pastoris ferments. MatTek full thickness skin tissues were treated with different $P$. pastoris ferments for $24 \mathrm{hr}$ and UVB irradiated at $225 \mathrm{~mJ} / \mathrm{cm}^{2}$ after the removal of preparations. Immediately after the UVB exposure the test materials were reapplied to the tissues and the tissues were incubated for 48 hours. Claudin 4 levels were tested using immunoblotting as described in the methods section. Data are presented as mean \pm SD.

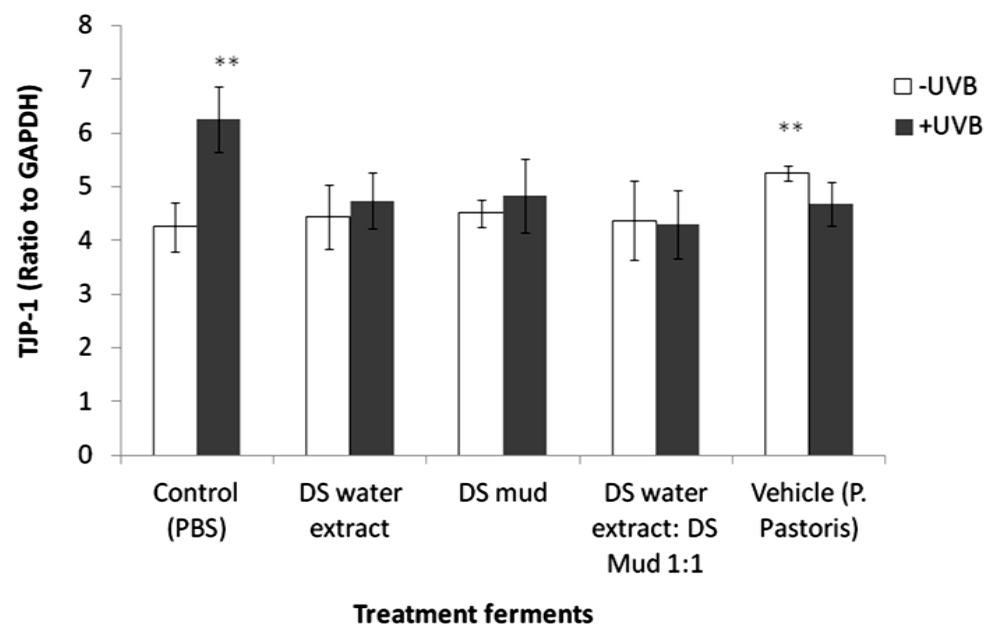

Figure 8. Skin tight junction protein 1 (TJP-1) levels following treatment with different $P$. pastoris ferments. MatTek full thickness skin tissues were treated with different $P$. pastoris ferments for $24 \mathrm{hr}$ and UVB irradiated at $225 \mathrm{~mJ} / \mathrm{cm}^{2}$ after the removal of preparations. Immediately after the UVB exposure the test materials were reapplied to the tissues and the tissues were incubated for 48 hours. TJP-1 levels were tested using immunoblotting as described in the methods section. Data are presented as mean \pm SD. ${ }^{* *} p<0.01$ irradiated vs. non-irradiated-control; vehicle vs. control-non-irradiated.

This study elucidated the effect of positive stress metabolites, derived from the yeast $P$. pastoris fermentation on human skin equivalents. This was performed by adding DS water extract and DS mud in small doses as nutrients supplements. P. pastoris was used as the microorganism mainly because its long history of exploitation in the pharmaceutical industry recognized as safe by the FDA, and only recently being explored for personal care applications.

In this study, the innovative concept of DS minerals supplementation as a positive stress to P. pastoris was investigated by testing the capability of yeast fermentation compounds to elicit beneficial effects on human skin 


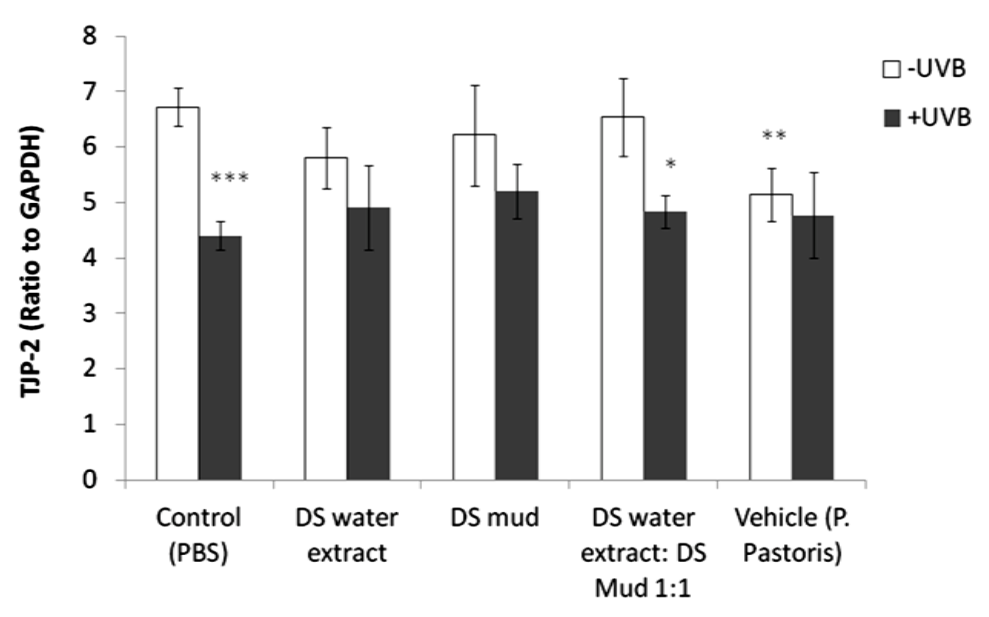

Treatment ferments

Figure 9. Skin tight junction protein 2 (TJP-2) levels following treatment with different $P$. pastoris ferments. MatTek full thickness skin tissues were treated with different $P$. pastoris ferments for $24 \mathrm{hr}$ and UVB irradiated at $225 \mathrm{~mJ} / \mathrm{cm}^{2}$ after the removal of preparations. Immediately after the UVB exposure the test materials were reapplied to the tissues and the tissues were incubated for 48 hours. TJP-2 levels were tested using immunoblotting as described in the methods section. Data are presented as mean \pm SD. ${ }^{* * *} p<0.001$ irradiated vs. non-irradiated-control; ${ }^{* *} p<0.01$ vehicle vs. control-non-irradiated; ${ }^{*} p<0.05$ irradiated vs. non-irradiated-DS water extract: DS mud 1:1.

using 3D human skin equivalents. The purpose was to test whether these compounds could serve as potent skincare actives. The main focus was on their effect on skin elasticity. Skin elasticity is crucial for skin appearance and supports lifting, firming and tightening and its properties can be reflected by different proteins related to extracellular matrix (ECM) and to dermal epidermal junctions (DEJ).

The observations clearly show that topical applications with fermentation compounds derived from P. pastoris exposed to DS minerals as a positive stress, have significant effects on the expression or activity of proteins related to skin elasticity. The major fermentation compounds' impact is on UVB-irradiated skin, where it can be seen that damage UVB-related proteins alteration effects can be attenuated.

Chronic exposure to UV solar radiation is usually the main environmental insult to human skin. Since UV exposure leads to skin photo-damage and consequently to skin aging, it has been selected as a stressor in this study.

Various protein biomarkers related to skin elasticity were tested in UVB-irradiated and non-irradiated skin equivalent samples following treatment with different $P$. pastoris ferments derived from exposure to DS water or mud.

From the results, it can be seen that skin exposure to UVB irradiation significantly led to the induction of different proteins related to ECM: elastin, fibulin-1 and LOX activity.

Elastin is one of the important components of elastic fibers in skin's ECM. Solar UV exposure leads to connective tissue damage that includes accumulation of abnormal elastic fibers. Studies show that UVB irradiation stimulates synthesis of elastin in human skin and experimental animals [15] [16] as well as of tropoelastin, a monomer precursor of elastin increased in human reconstituted skin [17].

Elastic fibers formation is catalyzed by LOX enzyme, which is crucial for the initiation of elastin formation by covalent cross-linking of tropoelastin monomers into elastin polymers. Therefore, it is expected that elastin formation will be associated with LOX activity.

Another protein which contributes to the elastic properties of connective tissue fibers and is involved with the process of fibrogenesis is the elastic fiber component Fibulin-1 [18].

The link among elastin, LOX and fibulin-1 in this study is emphasized due to their elevation following UVB irradiation, suggesting abnormal elastosis. However, pre-treatment with the different $P$. pastoris ferments has a photo-protective effect via eliminating or diminishing these 3 biomarkers UVB-induced elevation. 
Among all tested ferments, DS water extract ferment and DS mud ferment were the only ferments which significantly attenuated all three biomarkers that were induced following irradiation (Figures 2-4).

The mixture of DS water extract ferment and DS mud ferment reduced UVB-induced elastin and UVB-induced LOX, whilst the vehicle reduced only UVB-induced elastin. One proposed mode of action for reducing elevation of UVB-induced elastic fiber by DS elements-derived ferments can be via elastin cross-linking mechanism.

In non-irradiated skin only DS mud ferment significantly increased elastin level. However, this elevation was not accompanied by LOX activity elevation or fibulin level elevation. Here pre-treatment with DS mud ferment possesses a selective impact on elastin level, which differs between irradiated skin and non-irradiated skin. Thus, there is a possibility that the moderate increase in elastin level due to DS mud ferment application might occur due to another mechanism and may even have a positive effect on non-irradiated skin as no stress. This can be further elucidated by protein localization and structure.

Proteins of the matrix metalloproteinase (MMP) family are involved in the breakdown of ECM and during tissue remodeling in normal physiological processes as well as in pathological conditions. Exposure to UVB irradiation leads to inflammatory process affecting also on MMPs induction and might disrupt the balance towards abnormal ECM breakdown [19]. Among MMPs family MMP-3 can activate MMP-1, MMP-7 and MMP9 and therefore plays an important role in mediation of ECM degradation. In this study MMP-3 activity significantly increased as expected. All ferments reduced this UVB-induced activity (Figure 5). This result supports the protective effect against UVB-induced ECM degradation. MMP-3 degrades collagen, but also proteoglycans, fibronectin, laminin and elastin [20]. It has been demonstrated that in irradiated skin there is a strong link between enhancement in MMP-3 activity and collagen degradation [21]. The fact that not only elastin, but also other ECM protein serve as a target for MMP-3 can explain the elevation in elastin following irradiation despite the increase in MMP-3 activity. Moreover, it was reported that among MMP family members MMP-12 is the most active MMP against elastin [22]. This also explains that UVB irradiation may contribute to the accumulation of elastotic material and prevents the normal formation of elastic fibers in human skin and that different Metabiotics ${ }^{\mathrm{TM}}$ preparations can diminish this effect.

E-cadherin is the main cadherin in the human epidermis and controls epidermal adherent junctions. It is the major adhesion mediator between epidermal melanocytes and keratinocytes [23] [24]. Loss of E-cadherin appears to lead to malignant processes resulting in skin cancers such as melanoma and basal cell carcinoma [25] [26]. It has been shown previously that UVB irradiation induced the cleavage of E-cadherin in HaCaT cells [26]. In this study, UVB irradiation also led to a significant decrease in E-cadherin levels, suggesting damage to epidermal junctions (Figure 6). Application of DS water extract ferment and a mixture of DS water extract ferment and DS mud ferment prevented the decrease of E-cadherin following irradiation and hence, may provide a protection against this phenomenon. However, pre-treatment with DS mud ferment did not attenuate UVB-induced E-cadherin decrease, and led to a slight decrease in E-cadherin basal level and hence, might have a negative effect on this protein. Nevertheless, it is possible that in other concentrations this effect will be significantly reduced.

Tight junction proteins have been shown to be involved in barrier function of the skin by organizing the intracellular junctions. UVB irradiation is known to result in functional deterioration of tight junctions [27]. Tight junctions protein 1 (TJP-1) and tight junction protein 2 (TJP-2) expression was indeed altered following irradiation. TJP-1 level was elevated after irradiation, probably due an abnormal accumulation. Moreover, it has been shown that the location of TJP-1 alters after exposure to UVB [28]. All fermentation compounds prevented the UVB-induced over-expression of TJP-1 thus, might provide protection. TJP-2 level however, declined following irradiation pointing other damage mechanism to this protein by UVB and a complex damage to the TPs network. This decrease was not fully recovered by pre-treatment with fermentation compounds.

\section{Conclusion}

In conclusion, in this study, we managed to implement the positive stress concept by adding small amounts of Dead Sea minerals to the growth medium of the yeast $P$. pastoris and testing the generated fermentation compounds on human skin equivalents. The observations demonstrate that when these compounds are topically applied in a low concentration in-vivo (2\%) similarly to those of skincare actives, significant alterations in several proteins contributing to skin elasticity occur. Since a disruption in skin elasticity is a main phenomenon in aged or photo-damaged skin, these compounds are potent to serve as skincare actives. It is important to note that the 
in-vivo implications of an increase or decrease in a particular protein level are not always fully understood. However, these alterations provide a good basis for further elucidation of the altered protein localization and their structure as well as for in-vivo tests.

\section{References}

[1] Mattson, M.P. (2008) Hormesis Defined. Ageing Research Reviews, 7, 1-7. http://dx.doi.org/10.1016/j.arr.2007.08.007

[2] Mattson, M.P. and Cheng, A. (2006) Neurohormetic Phytochemicals: Low-Dose Toxins That Induce Adaptive Neuronal Stress Responses. Trends in Neurosciences, 29, 632-639. http://dx.doi.org/10.1016/j.tins.2006.09.001

[3] Sukenik, S., Buskila, D., Neumann, L., Kleiner-Baumgarten, A., Zimlichman, S. and Horowitz, J. (1990) Sulphur Bath and Mud Pack Treatment for Rheumatoid Arthritis at the Dead Sea Area. Annals of the Rheumatic Diseases, 49, 99102. http://dx.doi.org/10.1136/ard.49.2.99

[4] Moses, S.W., David, M., Goldhammer, E., Tal, A. and Sukenik, S. (2006) The Dead Sea, a Unique Natural Health Resort. Israel Medical Association Journal, 8, 483-488.

[5] Ma’or, Z., Henis, Y., Alon, Y., Orlov, E., Sorensen, K.B. and Oren, A. (2006) Antimicrobial Properties of Dead Sea Black Mineral Mud. International Journal of Dermatology, 45, 504-511. http://dx.doi.org/10.1111/j.1365-4632.2005.02621.x

[6] Even-Paz, Z. and Efron, D. (1996) The Dead Sea as a Spa Health Resort. Israel Journal of Medical Sciences, 32, S4S8.

[7] Hodak, E., Gottlieb, A.B., Segal, T., Politi, Y., Maron, L., Sulkes, J. and David, M. (2003) Climatotherapy at the Dead Sea Is a Remittive Therapy for Psoriasis: Combined Effects on Epidermal and Immunologic Activation. Journal of the American Academy of Dermatology, 49, 451-457. http://dx.doi.org/10.1067/S0190-9622(03)00916-2

[8] Proksch, E., Nissen, H.P., Bremgartner, M. and Urquhart, C. (2005) Bathing in a Magnesium-Rich Dead Sea Salt Solution Improves Skin Barrier Function, Enhances Skin Hydration, and Reduces Inflammation in Atopic Dry Skin. International Journal of Dermatology, 44, 151-157. http://dx.doi.org/10.1111/j.1365-4632.2005.02079.X

[9] Portugal-Cohen, M., Afriat-Staloff, I., Soroka, Y., Frusic-Zlotkin, M., Schlippe, G., Voss, W. and Ma’or, Z. (2014) Protective Effects of a Novel Preparation Consists of Concentrated Dead Sea Water and Natural Plants Extracts against Skin Photo-Damage. Journal of Cosmetics, Dermatological Sciences and Applications, 4, 7-15. http://dx.doi.org/10.4236/jcdsa.2014.41002

[10] Portugal-Cohen, M., Soroka, Y., Ma’or, Z., Oron, M., Zioni, T., Bregegere, F.M., Neuman, R., Kohen, R. and Milner, Y. (2009) Protective Effects of a Cream Containing Dead Sea Minerals against UVB-Induced Stress in Human Skin. Experimental Dermatology, 18, 781-788. http://dx.doi.org/10.1111/j.1600-0625.2009.00865.x

[11] Soroka, Y., Ma’or, Z., Leshem, Y., Verochovsky, L., Neuman, R., Bregegere, F.M. and Milner, Y. (2008) Aged Keratinocyte Phenotyping: Morphology, Biochemical Markers and Effects of Dead Sea Minerals. Experimental Gerontology, 43, 947-957. http://dx.doi.org/10.1016/j.exger.2008.08.003

[12] Wineman, E., Portugal-Cohen, M., Soroka, Y., Cohen, D., Schlippe, G., Voss, W., Brenner, S., Milner, Y., Hai, N. and Ma'or, Z. (2012) Photo-Damage Protective Effect of Two Facial Products, Containing a Unique Complex of Dead Sea Minerals and Himalayan Actives. Journal of Cosmetic Dermatology, 11, 183-192. http://dx.doi.org/10.1111/j.1473-2165.2012.00625.x

[13] Molnar, K. and Farkas, E. (2010) Current Results on Biological Activities of Lichen Secondary Metabolites: A Review. Zeitschrift für Naturforschung C, 65, 157-173. http://dx.doi.org/10.1515/znc-2010-3-401

[14] Takema, Y., Yorimoto, Y., Kawai, M. and Imokawa, G. (1994) Age-Related Changes in the Elastic Properties and Thickness of Human Facial Skin. British Journal of Dermatology, 131, 641-648. http://dx.doi.org/10.1111/j.1365-2133.1994.tb04975.x

[15] Schwartz, E., Feinberg, E., Lebwohl, M., Mariani, T.J. and Boyd, C.D. (1995) Ultraviolet Radiation Increases Tropoelastin Accumulation by a Post-Transcriptional Mechanism in Dermal Fibroblasts. Journal of Investigative Dermatology, 105, 65-69. http://dx.doi.org/10.1111/1523-1747.ep12312576

[16] Starcher, B., Pierce, R. and Hinek, A. (1999) UVB Irradiation Stimulates Deposition of New Elastic Fibers by Modified Epithelial Cells Surrounding the Hair Follicles and Sebaceous Glands in Mice. Journal of Investigative Dermatology, 112, 450-455. http://dx.doi.org/10.1046/j.1523-1747.1999.00553.X

[17] Seo, J.Y., Lee, S.H., Youn, C.S., Choi, H.R., Rhie, G.E., Cho, K.H., Kim, K.H., Park, K.C., Eun, H.C. and Chung, J.H. (2001) Ultraviolet Radiation Increases Tropoelastin mRNA Expression in the Epidermis of Human Skin in Vivo. Journal of Investigative Dermatology, 116, 915-919. http://dx.doi.org/10.1046/j.1523-1747.2001.01358.x

[18] Roark, E.F., Keene, D.R., Haudenschild, C.C., Godyna, S., Little, C.D. and Argraves, W.S. (1995) The Association of Human Fibulin-1 with Elastic Fibers: An Immunohistological, Ultrastructural, and RNA Study. Journal of Histochemi- 
stry \& Cytochemistry, 43, 401-411. http://dx.doi.org/10.1177/43.4.7534784

[19] Cox, T.R. and Erler, J.T. (2011) Remodeling and Homeostasis of the Extracellular Matrix: Implications for Fibrotic Diseases and Cancer. Disease Models \& Mechanisms, 4, 165-178. http://dx.doi.org/10.1242/dmm.004077

[20] Quan, T., Qin, Z., Xia, W., Shao, Y., Voorhees, J.J. and Fisher, G.J. (2009) Matrix-Degrading Metalloproteinases in Photoaging. Journal of Investigative Dermatology Symposium Proceedings, 14, 20-24. http://dx.doi.org/10.1038/jidsymp.2009.8

[21] Rittie, L. and Fisher, G.J. (2002) UV-Light-Induced Signal Cascades and Skin Aging. Ageing Research Reviews, 1, 705-720. http://dx.doi.org/10.1016/S1568-1637(02)00024-7

[22] Chen, Z., Seo, J.Y., Kim, Y.K., Lee, S.R., Kim, K.H., Cho, K.H., Eun, H.C. and Chung, J.H. (2005) Heat Modulation of Tropoelastin, Fibrillin-1, and Matrix Metalloproteinase-12 in Human Skin in Vivo. Journal of Investigative Dermatology, 124, 70-78. http://dx.doi.org/10.1111/j.0022-202X.2004.23550.X

[23] Furukawa, F., Fujii, K., Horiguchi, Y., Matsuyoshi, N., Fujita, M., Toda, K., Imamura, S., Wakita, H., Shirahama, S. and Takigawa, M. (1997) Roles of E- and P-Cadherin in the Human Skin. Microscopy Research and Technique, 38, 343-352. http://dx.doi.org/10.1002/(SICI)1097-0029(19970815)38:4<343::AID-JEMT2>3.0.CO;2-K

[24] Gruss, C. and Herlyn, M. (2001) Role of Cadherins and Matrixins in Melanoma. Current Opinion in Oncology, 13, 117-123. http://dx.doi.org/10.1097/00001622-200103000-00006

[25] Fuller, L.C., Allen, M.H., Montesu, M., Barker, J.N. and Macdonald, D.M. (1996) Expression of E-Cadherin in Human Epidermal Non-Melanoma Cutaneous Tumours. British Journal of Dermatology, 134, 28-32. http://dx.doi.org/10.1111/j.1365-2133.1996.tb07835.x

[26] Hung, C.F., Chiang, H.S., Lo, H.M., Jian, J.S. and Wu, W.B. (2006) E-Cadherin and Its Downstream Catenins Are Proteolytically Cleaved in Human HaCaT Keratinocytes Exposed to UVB. Experimental Dermatology, 15, 315-321. http://dx.doi.org/10.1111/j.0906-6705.2006.00411.x

[27] Yuki, T., Hachiya, A., Kusaka, A., Sriwiriyanont, P., Visscher, M.O., Morita, K., Muto, M., Miyachi, Y., Sugiyama, Y. and Inoue, S. (2011) Characterization of Tight Junctions and Their Disruption by UVB in Human Epidermis and Cultured Keratinocytes. Journal of Investigative Dermatology, 131, 744-752. http://dx.doi.org/10.1038/jid.2010.385

[28] Yamamoto, T., Kurasawa, M., Hattori, T., Maeda, T., Nakano, H. and Sasaki, H. (2008) Relationship between Expression of Tight Junction-Related Molecules and Perturbed Epidermal Barrier Function in UVB-Irradiated Hairless Mice. Archives of Dermatological Research, 300, 61-68. http://dx.doi.org/10.1007/s00403-007-0817-y 
Scientific Research Publishing (SCIRP) is one of the largest Open Access journal publishers. It is currently publishing more than 200 open access, online, peer-reviewed journals covering a wide range of academic disciplines. SCIRP serves the worldwide academic communities and contributes to the progress and application of science with its publication.

Other selected journals from SCIRP are listed as below. Submit your manuscript to us via either submit@scirp.org or Online Submission Portal.
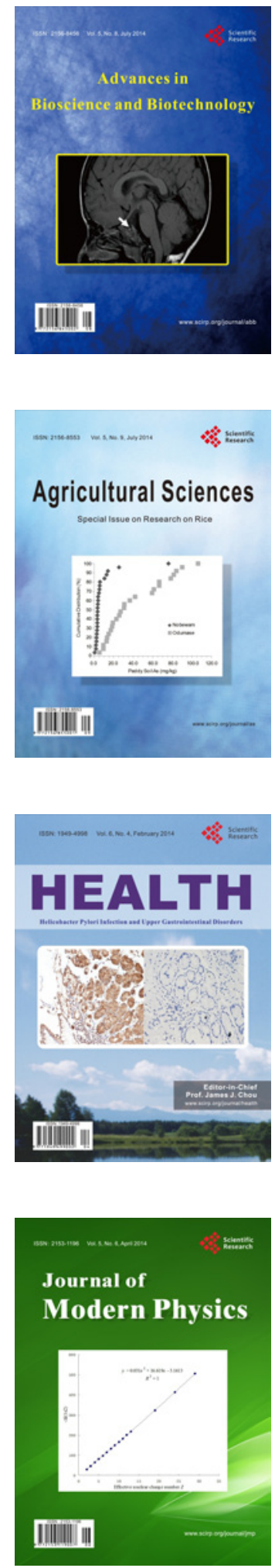
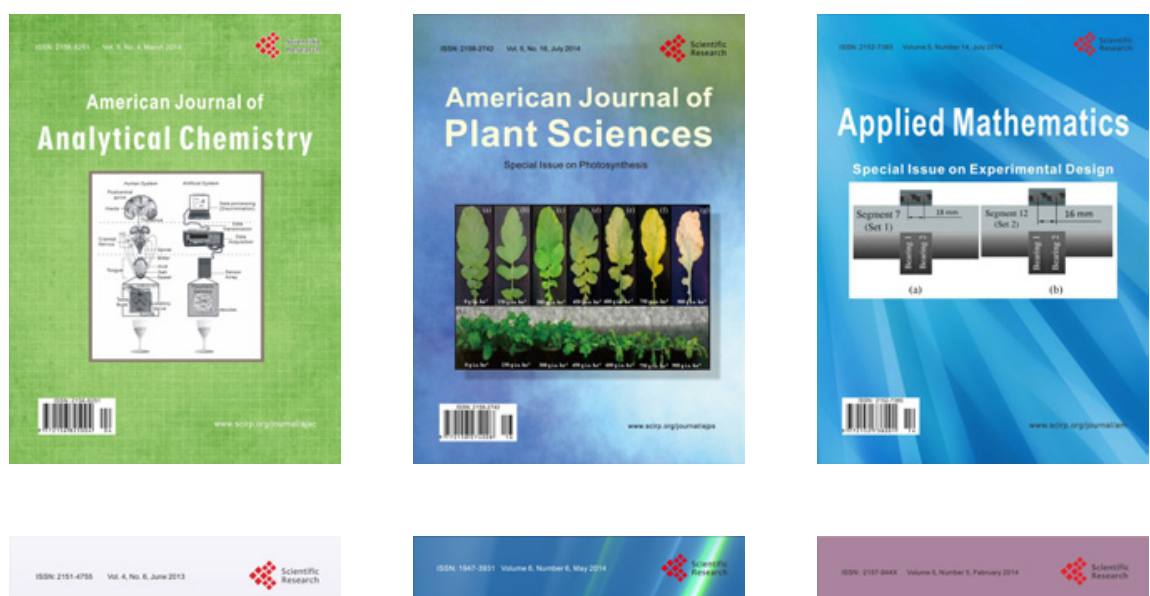

Creative Education
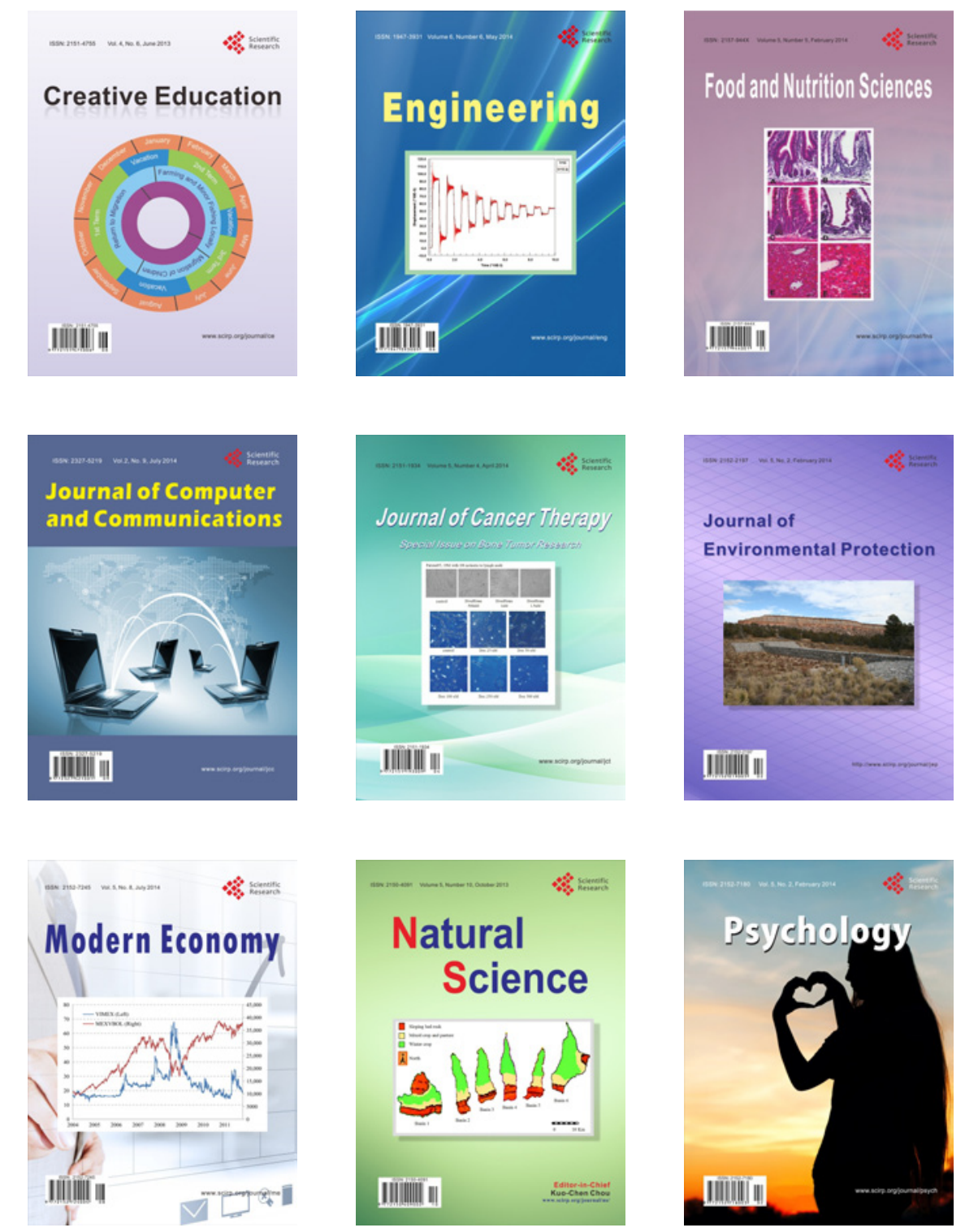